\title{
AN OBSERVATIONAL STUDY COMPARING SITAGLIPTIN TO METFORMIN AS A INITIAL MONOTHERAPY IN TYPE 2 DIABETES MELLITUS PATIENTS
}

\author{
Mohd. Riyaz ${ }^{1}$, Imran², Rinu Manuel ${ }^{3}$, Nidhisha K. Joseph ${ }^{4}$
}

\section{HOW TO CITE THIS ARTICLE:}

Mohd. Riyaz, Imran, Rinu Manuel, Nidhisha K. Joseph. "An Observational Study Comparing Sitagliptin to Metformin as an Initial Monotherapy in Type 2 Diabetes Mellitus Patients". Journal of Evolution of Medical and Dental Sciences 2014; Vol. 3, Issue 07, February 17; Page: 1698-1703, DOI: 10.14260/jemds/2014/2044

ABSTRACT: AIM: An Observational study Comparing sitagliptin to metformin as an initial monotherapy in type 2 diabetes mellitus patients. METHOD: In this observational study we have selected 100 patients which divided into two groups; first group was on sitagliptin and second group was on metformin. The criterion for inclusion in the study was 1) Age group of 18 - 78 years 2) HbA1c of 6.5 - 9.0\%. Group A was started on sitagliptin 50 to $100 \mathrm{mg}$. Group B patients were started on Metformin, dosage of $500 \mathrm{mg}$ once a day and the dose was increased to a of maximum of $2000 \mathrm{mg}$; and this study was done for 12 weeks. RESULTS AND STATISTICAL ANALYSIS: Group A comprises 50 patients whose baseline FBS, PPBS, HbA1C was determined, Group B comprises 50 patients whose baseline FBS, PPBS, HbA1C was determined. The results of HbA1c obtained before and after the sitagliptin and metformin therapy was analyzed using SPSS V 20 software using paired test and chi square analysis. It was observed that the mean baseline hba1c in the group A patients was $7.8 \%$ and in group B was $7.95 \%$ whereas the mean HbA1c after 12 weeks of therapy was $7.44 \%$ and $7.73 \%$ respectively in the Group A and B. There was a significant decrease in Hba1c in both groups with $t$ (49) value Group A (7.89, p <0.001) Group B (3.48, p 0.001). There was a significantly positive correlation $(\mathrm{p}<0.001)$ in two groups, $A(r=0.903)$ having higher positive correlation than group $B(r=$ 0.748), whereas the mean difference in Hba1c was higher for group A (0.36) compared to group B $(0$, 21) suggesting a greater decrease in Hba1c with sitagliptin compared to metformin, as the mean difference was significant the effect size (d) was calculated it was observed that the effect size for group A was $(\mathrm{d}=1.16)$ larger than group $\mathrm{B}(\mathrm{d}=0.49)$. Occurrence of Various symptoms such as nausea, headache and diarrhoea were seen in two groups and it was observed that nausea was greater in group $A(16 \%)$ compared to $B(8 \%)$, the increase was observed to $b$ non-significant at $p=$ 0.35 , headache was greater in group $B(10 \%)$ compared to $A(5 \%)$, the increase was observed to $b$ non-significant at $p=1.0$, Diarrhoea was greater in group B (18\%) compared to A (2\%), the increase was observed to $\mathrm{b}$ significant at $\mathrm{p}=0.016$ with a likelihood ratio of 8.0. CONCLUSION: In this 12 week study sitagliptin was not inferior compared to metformin in controlling the Hba1C in patients with type 2 diabetes mellitus. Both the group; patients well tolerated and the incidence of gastrointestinal adverse effects were lower in the patients on sitagliptin.

KEYWORDS: sitagliptin; metformin, biguanides, dipeptidyl peptidase-4, incretins.

INTRODUCTION: Sitagliptin is an oral antihyperglycaemic agent. It is a selective DPP-4 (dipeptidyl peptidase-4) inhibitor and is indicated for the treatment of type 2 diabetes mellitus. In clinical trials, sitagliptin monotherapy has shown to improve glycemic control and $\beta$-cell function, with low risk of hypoglycemia, gastrointestinal side effects, and no weight gain ${ }^{1,2}$. 
Metformin is recommended as monotherapy for treatment of type 2 diabetes mellitus because of its efficacy and long-term use ${ }^{3}$.However, the common side effects are gastrointestinal intolerance and risk of lactic acidosis in low perfusion states and Renal Failure ${ }^{3-6}$.

In trials (Aschner et al; Raz I et al), treatment with once-daily 100mg sitagliptin significantly reduced $\mathrm{HbA} 1 \mathrm{c}$ by $0.7 \%$ compared with placebo in patients with mild-to-moderate hyperglycemia (mean base-line $\mathrm{HbA}_{1 \mathrm{c}} \sim 8 \%$ ) . The proportion of patients with $\mathrm{HbA} 1 \mathrm{c}<7 \%$ was approximately $40 \%$ in the sitagliptin group com-pared with approximately $16 \%$ in the placebo groups in these studies ${ }^{6-}$ 12 .

In different clinical situations, it was considered that both sitagliptin and Metformin as initial monotherapy is effective in patients with type 2 diabetes. This 12 week study compared the lowering of HbA1c (efficacy) and safety of sitagliptin monotherapy and Metformin monotherapy, in type 2 diabetes mellitus ${ }^{11,12}$.

\section{METHODS:}

\section{Inclusion Criteria:}

1) Men and women with type 2 diabetes (18-78 years of age)

2) $\mathrm{HbA}_{1 \mathrm{c}} 6.5-9.0 \%$ were eligible to participate in the study

\section{Exclusion Criteria:}

1) Type 1 diabetes,

2 ) Fasting plasma glucose $(\mathrm{FPG})<120 \mathrm{mg} / \mathrm{dl}$ or $>250 \mathrm{mg} / \mathrm{dl}$,

3) Unstable cardiac disease,

4 ) Renal impairment (creatinine $\geq 1.4 \mathrm{mg} / \mathrm{dl}$ for males or $\geq 1.3 \mathrm{mg} / \mathrm{dl}$ for females or creatinine clearance $<60 \mathrm{ml} / \mathrm{min}$ ),

5) Elevated alanine aminotransferase, aspartate aminotransferase,

6) Creatine phosphokinase (more than 2 times upper limit of normal) Triglycerides $>600$ $\mathrm{mg} / \mathrm{dl}$.

Patients were counseled regarding diet and exercise for the duration of the study.

STUDY DESIGN: This Observational study was conducted at Esani Diabetes and multi-specialty center to assess similarity of efficacy of a new agent to a standard treatment. In the present study 100 known diabetic patients were selected from Esani diabetes and Multispecialty center, the patients were randomly divided into two groups, group A comprised 50 patients whose baseline FBS, PPBS, HbA1c was determined and the patients were put on sitagliptin 50 - $100 \mathrm{mg}$ per day, Group B comprised 50 patients whose baseline FBS, PPBS, HbA1c was determined and the patients were put on metformin 500 - $2000 \mathrm{mg}$ per day to achieve control of blood sugars and repeat testing for HbA1c was done after 12 weeks, patients were monitored closely with proper diabetic diet counseling; for ensuring the compliance to diet, drug and exercise.

Metformin $500 \mathrm{mg}$ was started as once a day daily dose and titrated u p wards to $500 \mathrm{mg}$ twice daily over a maximum 5-week period. Down-titration of metformin was permitted for intolerance to a minimum allowed dose of $1000 \mathrm{mg} /$ day. Patients treated with were analyzed as a single group, regardless of final dose. 
Study Evaluations: Efficacy Assessments: After an overnight fast of $\geq 12 \mathrm{~h}$ in duration, blood samples were collected for HbA1c estimation.

Other endpoints included the proportions of patients with $\mathrm{HbA} 1 \mathrm{c}<7$ or $<6.5 \%$,

- FBG,

- lipid parameters which includes triglycerides (TG); total cholesterol (TC); LDL-C; HDL-C,

- Sr. Creatinine

Safety Assessments. Data were collected on,

- clinical and laboratory adverse experiences (AEs),

- physical examinations,

- body weight,

- vital signs and

- Electrocardiograms (ECGs) at predefined time points.

Patients were counseled with regard to the symptoms, finger stick evaluation and treatment of hypoglycemia; those experiencing symptoms consistent with hypoglycemia were to obtain finger stick glucose, value and keep a record in the logbook.

History of adverse effect were recorded such as symptomatic hypoglycemia, (headache; tremors) and the gastrointestinal adverse Effects such as diarrhoea, nausea, abdominal pain and vomiting.

RESULTS AND STATISTICAL ANALYSIS: In the present study 100 known diabetic patients were selected from esani diabetes and Multispecialty center, the patients were randomly divided into two groups, Group A comprised 50 patients whose baseline FBS, PPBS, HbA1c was determined and the patients were put on sitagliptin 50 - 100 mg per day, Group B comprised 50 patients whose baseline FBS, PPBS, mg per day to HbA1c was determined and the patients were put on metformin $500-2000$ achieve control of blood sugars and repeat testing for $\mathrm{HbA1c}$ was done after 12 weeks, patients were also monitored for presence of any symptoms such as nausea, headache and diarrhoea.

The results of HbA1c obtained before and after the sitagliptin and metformin therapy was analyzed using SPSS V 20 software using paired t test and chi square analysis.

It was observed that the mean baseline HBA1c in the Group A patients was $7.8 \%$ and in Group B was 7.95\%, whereas the mean HbA1c after 12 weeks of therapy was $7.44 \%$ and $7.73 \%$ respectively in the Group A and B. There was a significant decrease in HBA1c in both groups with $t$ (49) value, Group A (7.89, p <0.001) Group B (3.48, p 0.001).

There was a significantly positive correlation $(\mathrm{p}<0.001)$ in two groups, group $A(r=0.903)$ having higher positive correlation than group $B(r=0.748)$, whereas the mean difference in HbA1c was higher for group A $(0.36)$ compared to group B $(0,21)$ suggesting a greater decrease in HbA1c with sitagliptin compared to metformin, as the mean difference was significant the effect size (d) was calculated. It was observed that the effect size for group A was $(d=1.16)$ larger than group $B(d=$ 0.49).

Occurrence of Various symptoms such as nausea, headache and diarrhoea were seen in both groups and it was observed that nausea was greater in group A (16\%) compared to B (8\%), the increase was observed to be non-significant at $p=0.35$. Headache was greater in group $B(10 \%)$ compared to A (5\%), the increase was observed to be non-significant at $p=1.0$. Diarrhoea was 
greater in group B (18\%) compared to group A (2\%), the increase was observed to be significant at p $=0.016$ with a likelihood ratio of 8.0.

DISCUSSION: DPP-4 inhibitors (Sitagliptin) could be used as initial therapy as they have glucosedependent $\beta$-cell sensitizer activity. DPP-4 inhibitors are well tolerated and have a low risk of hypoglycemia or weight gain.1,2

Metformin is widely used as an oral agent for the treatment of type 2 diabetes mellitus. It is recommended as an initial monotherapy.

Metformin helps in weight loss and has a record of efficacy and safety, with a lowest risk for hypoglycemia. ${ }^{3,6}$

Insulin secretagogues, such as sulfonylurea agents, are associated with weight gain and hypoglycemia, $\alpha$-glucosidase inhibitor is associated with some gastrointestinal side effects.

Sitagliptin, a DPP-4 inhibitor is approved for the treatment of type 2 diabetes since 2006 in USA \& in 2007 by EU. Comparison of the safety and efficacy of sitagliptin to metformin in patients who are not at their $\mathrm{HbA}_{1} \mathrm{c}$ goal and require treatment with an oral antihyperglycaemic agent was the objective of this study. ${ }^{1,2,6-8}$

The criterion for this observational study was patients having an $\mathrm{HbA1c}$ value between 6.5 and $9.0 \%$, which was lower than other trials with antihyperglycaemic agents. This range was chosen to compare sitagliptin with metformin in clinical scenarios in which these agents would be used as monotherapies.

International Diabetes Federation (IDF) and the American Association of Clinical Endocrinologists (AACEs), suggest that an HbA1c $\leq 6.5 \%$ is the appropriate target for patients with type 2 diabetes and reduces the risk for microvascular complications. ${ }^{3}$

It was observed that the mean baseline HBA1c in the Group A patients was $7.8 \%$ and in Group B was 7.95\% whereas the mean HbA1c after 12 weeks of therapy was $7.44 \%$ and $7.73 \%$ respectively in the Group A and B. There was a significant decrease in HBA1c in both groups with $t$ (49) value Group A (7.89, p <0.001) Group B (3.48, p< 0.001).

There was a significantly positive correlation $(\mathrm{p}<0.001)$ in two groups, $A(r=0.903)$ having higher positive correlation than group $B(r=0.748)$, whereas the mean difference in HbA1c was higher for group A (0.36) compared to group B (0.21) suggesting a greater decrease in HbA1c with sitagliptin compared to metformin, as the mean difference was significant the effect size (d) was calculated. It was observed that the effect size for group A was $(d=1.16)$ larger than group $B(d=$ 0.49).

Occurrence of various symptoms such as nausea, headache and diarrhoea were seen in both groups and it was observed that nausea was greater in group A (16\%) compared to B (8\%), the increase was observed to be non-significant at $p=0.35$. Headache was greater in group B (10\%) compared to group A (5\%), the increase was observed to be non-significant at $p=1.0$. Diarrhoea was greater in group B (18\%) compared to A $(2 \%)$, the increase was observed to be significant at $\mathrm{p}=$ 0.016 with a likelihood ratio of 8.0.

The safety and efficacy of sitagliptin was compared to metformin in patients with type 2 diabetes who were having mild-to-moderate hyperglycemia. At study end, majority of the patients in group B were on metformin $2000 \mathrm{mg} /$ day because Metformin provided good efficacy in a doseranging from $500-2500 \mathrm{mg}$ per day. There was a small but statistically significant difference in 
reduction of $\mathrm{HbA} 1 \mathrm{c}$ between the two groups i.e. (0.14\%), sitagliptin was not inferior compared to metformin for the control of HbA1c.

Bloomgarden et al, have shown that different oral antihyperglycaemic agents have similar efficacy when the data are corrected for differences in baseline HbA1c values. ${ }^{8}$ As the FPG level Changes in both the treatment groups and has a marked decrease in first 6 weeks and levels then remained stable for the duration of the study. The impact of the reduction in FPG levels was moderately greater for metformin group compared to the sitagliptin group of patients. The difference between treatments may be related to the mechanisms of both agents, patients on metformin have the FPG reduction could be due to its primary mechanism of action i.e. inhibition of hepatic glucose production. ${ }^{5,8,10-12}$ The FPG reduction by sitagliptin is because of the incretin-mediated effects on insulin (stimulate release) or glucagon (inhibit secretion).

Both are having glucose- dependent action and which begins to dissipate as the blood glucose levels approach normal levels ${ }^{12}$. Hence, in this study patients who had low mean baseline FPG ( $\sim 142$ $\mathrm{mg} / \mathrm{dl}$ ), the glucose-dependent effects of sitagliptin on FPG were likely diminished as sitagliptin reduced FPG to near-normal levels (mean FPG at nadir was $\sim 130 \mathrm{mg} / \mathrm{dl}$ ). We found, that there was a lower incidence of gastrointestinal- related side effects with sitagliptin and a lower incidence of hypoglycemia in both the groups. In the context of equivalent glycemic improvement, body weight was reduced more with metformin than with sitagliptin. $1,2,5,6$

Recent data indicate that early and aggressive treatment in patient with type 2 diabetes mellitus reduces the risk of long-term micro-and macro-vascular complications.

CONCLUSION: In summary, either sitagliptin or metformin monotherapy helped in improving glycemic control as measured by HbA1c; in the treatment of patients with type 2 diabetes mellitus. Both the group of the drugs was well tolerated, and the incidence of gastrointestinal-related adverse effects was lower, but weight loss was less in the group of patients on sitagliptin. The result of this study provides the data for the use of sitagliptin as initial monotherapy for type 2 diabetes mellitus patients.

\section{REFERENCES:}

1. Aschner $\mathrm{P}$ et al. Effect of the dipeptidyl peptidase-4 inhibitor sitagliptin as monotherapy on glycemic control in patients with type 2 diabetes. 2006 Diabetes Care; 29: 2632 - 2637.

2. Raz I, Hanefeld M, Xu L, Caria C, Williams-Herman D, Khatami H. Efficacy and safety of the dipeptidyl peptidase-4 inhibitor sitagliptin as monotherapy in patients with type 2 diabetes mellitus. Diabetologia 2006; 49: 2564 - 2571.

3. Nathan DM, Buse JB, Davidson MB et al. Management of hyperglycemia in type 2 diabetes: a consensus algorithm for the initiation and adjustment of therapy: a consensus statement from the ADA and the EAS. 2006 Diabetes Care; 29: 1963 - 1972.

4. Scarpello JH, Howlett HC. Metformin therapy and clinical uses. Diab Vasc Dis Res 2008; $5: 157$ 167.

5. Green J, Feinglos M. New combination treatments in the management of diabetes: focus on sitagliptin-metformin. Vasc Health Risk Manag 2008; 4: 743 - 751. 
6. Goldstein BJ, Feinglos MN, Lunceford JK, Johnson J, Williams-Herman DE. Effect of initial combination therapy with sitagliptin, a dipeptidyl peptidase-4 inhibitor, and metformin on glycemic control in patients with type 2 diabetes. Diabetes Care 2007; 30: 1979 - 1987.

7. Karasik A, Aschner P, Katzeff H, Davies MJ, Stein PP. Sitagliptin, a DPP-4 inhibitor for the treatment of patients with type 2 diabetes: a review of recent clinical trials. Curr Med Res Opin 2008; 24: 489 - 496.

8. Bloomgarden ZT, Dodis R, Viscoli CM, Holmboe ES, Inzucchi SE. Lower baseline glycemia reduces apparent oral agent glucose-lowering efficacy: a meta-regression analysis. Diabetes Care 2006; 29: 2137 - 2139.

9. Drucker DJ, Nauck MA. The incretin system: glucagon-like peptide-1 receptor agonists and dipeptidyl peptidase-4 inhibitors in type 2 diabetes. Lancet 2006; 368: 1696 - 1705.

10. Hanefeld M, Herman GA, Wu M, Mickel C, Sanchez M, Stein PP. Once-daily sitagliptin, a dipeptidyl peptidase-4 inhibitor, for the treatment of patients with type 2 diabetes. Curr Med Res Opin 2007; 23: 1329 - 1339.

11. Nonaka K, Kakikawa T, Sato A. et al. Efficacy and safety of sitagliptin monotherapy in Japanese patients with type 2 diabetes. Diabetes Res Clin Pract 2008; 79: 291 - 298.

12. Williams-Herman D, Johnson J, Teng R et al. Efficacy and safety of initial combination therapy with sitagliptin and metformin in patients with type 2 diabetes: a 54-week study. Curr Med Res Opin 2009; 25: $569-583$.

\section{AUTHORS:}

1. Mohd. Riyaz

2. Imran

3. Rinu Manuel

4. Nidhisha K. Joseph

\section{PARTICULARS OF CONTRIBUTORS:}

1. Assistant Professor, Department of Medicine, M.N.R. Medical College, Sangareddy, Andhra Pradesh.

2. Consultant Physician, Esani Diabetes \& Multispeciality Centre, Tolichowki, Hyderabad, Andhra Pradesh.

3. Senior Diabetic Eudcator, Esani Diabetes \& Multispeciality Centre, Tolichowki, Hyderabad, Andhra Pradesh.
4. Diabetic Educator, Esani Diabetes \& Multispeciality Centre, Tolichowki, Hyderabad, Andhra Pradesh.

\section{NAME ADDRESS EMAIL ID OF THE CORRESPONDING AUTHOR:}

Dr. Mohd. Riyaz, 11-5-337/2, Red Hills, Hyderabad, Andhra Pradesh - 500004.

E-mail: riyazesani@hotmail.com

Date of Submission: 24/01/2014. Date of Peer Review: 25/01/2014. Date of Acceptance: 05/02/2014. Date of Publishing: 13/02/2014. 